\title{
Single-machine rework rescheduling to minimize maximum waiting-times with fixed sequence of jobs and ready times
}

\author{
Yandong Guo a, b, Min Huang *, a, Qing Wang a, V. Jorge Leon c \\ a College of Information Science and Engineering, Northeastern University; State Key Laboratory of Synthetical \\ Automation for Process Industries (Northeastern University), Shenyang, Liaoning, 110819, China \\ b School of Mathematics and Physics, Bohai University Jinzhou, Liaoning, China, 121013 \\ ${ }^{c}$ Department of Engineering Technology and Industrial Distribution and Department of Industrial and Systems \\ Engineering, Texas A\&M University, College Station, Texas 77843-3367 \\ * Corresponding author. E-mail addresses: mhuang@mail.neu.edu.cn
}

\begin{abstract}
This paper studies a single machine rescheduling problem in which a set of newly arrived rework jobs must be inserted into an existing schedule. The original jobs have ready times, and the objective is to minimize the maximum waiting time of any job in the final schedule. The problem is demonstrated to be NP-Hard. Properties of the optimal solution are developed, enabling the development of a pseudo-polynomial algorithm for a special case and a heuristic algorithm for a general problem. A heuristic procedure is developed, and its worst case performance is derived. Experimental results suggest that the heuristic yields optimal and close-to-optimal solutions to problems similar to those present in practice.
\end{abstract}

Key words: Rescheduling; rework jobs; waiting time; heuristic algorithm; NP-hard; single machine. 


\title{
Single-machine rework rescheduling to minimize maximum waiting-times with fixed sequence of jobs and ready times
}

\begin{abstract}
This paper studies a single machine rescheduling problem in which a set of newly arrived rework jobs must be inserted into an existing schedule. The original jobs have ready times, and the objective is to minimize the maximum waiting time of any job in the final schedule. The problem is demonstrated to be NP-Hard. Properties of the optimal solution are developed, enabling the development of a pseudo-polynomial algorithm for a special case and a heuristic algorithm for a general problem. A heuristic procedure is developed, and its worst case performance is derived. Experimental results suggest that the heuristic yields optimal and close-to-optimal solutions to problems similar to those present in practice.
\end{abstract}

Key words: Rescheduling; rework jobs; waiting time; heuristic algorithm; NP-hard; single machine. 


\section{Introduction}

Production environments are dynamic and are often affected by unforeseen events, such as rush orders, changes in job release times or priorities, order cancellations, processing delays, rework, machine breakdowns, shortages of raw materials, personnel problems, equipment downtimes, and tool breakdowns. These disruptions can make a-priori production schedules obsolete soon after being released for execution. Rescheduling addresses the problem of adapting the disrupted a-priori schedule such that it recovers its viability for execution. Leon (1991) and Wu et al. (1992) contributed to early work in rescheduling in the context of job-shops prone to disruptions caused by unforeseen events. The taxonomy of rescheduling literature can be found in Vieira et al. (2003) and Herrmann et al. (2006).

The rescheduling problem in this paper is inspired by a case involving a company in northeastern China that manufactures products completely made of quartz. The factory under consideration makes trays, magazines, and other material handling tools that will be in direct contact with the silicon wafers in a semiconductor manufacturing facility. While quartz is extremely stable in corrosive environments and possesses excellent properties for use in clean-rooms, it is also fragile. Due to the latter, quartz products can be damaged (e.g., cracks, chips, and breaks) during processing and handling in a manufacturing facility. Damaged products are either discarded or reworked depending on the gravity of the defect; i.e., a damaged product is reworked only if its processing time is shorter than a given threshold value. The scheduling problem of interest occurs at a reheating oven, where both the rework stream and the regular production streams meet. It is important that the sequence planned for regular production is not disrupted to avoid damage of the product, as well as other components that will join it downstream, due to the additional material handling caused by a possible change in sequence. This paper studies a single machine rescheduling problem to decide where to insert the rework jobs in a given sequence of regular jobs to minimize maximum waiting time.

Unal et al. (1997) studied a similar problem, considering sequence dependent family setup times. With sequence dependent family setup times, these researchers attempted to minimize deviations of the jobs from their original due-dates while minimizing the makespan of the new jobs. The authors proposed a polynomial-time procedure to determine whether there exists a solution in which no additional setups are required while satisfying the deadlines and maintaining the original schedule. These researchers also demonstrated that that the problem of minimizing total weighted completion time is NP-hard in general but is polynomial in the case where processing times are unitary.

Hall et al. (2004) proposed polynomial time algorithms and showed the NP-hardness of various single-machine rescheduling problems. In each problem, the objective function contains a component that is a function of the completion time and a second component that captures costs associated with disruptions. These researchers defined a disruption as the degree of changes between the original and rescheduling schedules, i.e., sequence disruptions and time disruptions. The authors also considered cases where the disruption cost is considered as a constraint. Later, Hall et al. (2007) proposed a branch-and-bound algorithm for a single-machine rescheduling problem with multiple disruptions. In a similar line of work, Yuan et al. (2007) considered a single machine problem to minimize the makespan 
subject to the constraint of a disruption criterion bounded by a given value, release times, and the assumption that the original sequence is optimal. These researchers demonstrated that the problem is NP-hard for the cases where the disruption criterion is either the maximum time-disruption or the total of time-disruption. The authors also demonstrated that the case where the disruption criterion is the maximum sequence disruption can be solved in polynomial time. Hoogeveen et al. (2012) provided polynomial-time algorithms or NP-hardness proofs for the single machine problem given an optimal schedule that minimizes total setup time. The new jobs must be incorporated within the original optimal schedule, resulting in a disruption cost. The rescheduling problem aims at minimizing the disruption cost and the total setup times in the overall schedule.

Yang (2007) considered a single-machine problem in which each new job has a processing time that can be compressed at the price of an extra cost. The cost may increase as the processing time of the new job decreases. The aim is to schedule the new jobs and to determine their actual processing times to minimize a convex combination of the total costs of compression, a disruption criterion and a classic scheduling criterion. Yang showed that in the case of minimizing total completion-times, the problem can be solved in polynomial time, while the case that considers total tardiness is NP-hard. In a similar line of work, Zhao et al. (2010) considered the case of deteriorating jobs, in which processing times depend on their starting time; specifically, a later start leads to a longer processing time. The total completion time problem, subject to given bounds on the maximum positional or completion time disruptions, is shown to be polynomial under certain restrictions on the processing times.

The rest of the paper is organized as follows: Section 2 develops the model and problem formulation. Section 3 presents the complexity analysis of the problem. Section 4 solves a special case of the problem using a pseudo-polynomial algorithm. Section 5 studies the general problem, develops a heuristic, and derives its optimal and worse-case performance for certain cases. The section also derives conditions for which the heuristic yields optimal solutions. Section 6 contains the experimental study for the evaluation of the performance of the heuristic. Finally, Section 7 presents concluding remarks and suggestions for future research.

\section{Model and Problem Formulation}

Let $J_{O}=\left\{1, \ldots, n_{O}\right\}$ be the set of original jobs that are scheduled for processing non-preemptively on a single machine. Let $J_{R}=\left\{n_{O}+1, \ldots, n_{O}+n_{R}\right\}$ be a set of new rework jobs, which will also be processed without preemption. Let $J=J_{O} \cup J_{N}$ and $n=|J|=n_{O}+n_{R}$. Let $p_{j}$ and $r_{j}$ denote the processing time and ready-time of job $j$ for $j \in J$, respectively. Let $v$ denote the original schedule of the jobs in $J_{O}$. No restriction is imposed on the optimality of the original schedule $v$. The optimal and feasible schedule for all jobs in $J$ are denoted as $\sigma^{*}$ and $\sigma$, respectively. The decision variables are the start-times in schedule $\sigma, s_{j}(\sigma)$, for $j \in J$. In the paper, all schedules involved are active; an active schedule is defined as a schedule such that no job can be scheduled earlier without delaying another one.

For clarity, variables that depend on a particular schedule will indicate this explicitly; e.g., $C_{j}(\pi)$ and $w_{j}(\pi)=s_{j}(\pi)-r_{j}=C_{j}(\pi)-p_{j}-r_{j}$ indicate the completion time of job $j$ and the waiting time of 
job $j$ in schedule $\pi$, respectively, for $j \in J, \pi \in\left\{v, \sigma, \sigma^{*}\right\}$; the waiting time of the original jobs are bounded by a value $K$; the delay time of the original job $j$ equals to $s_{j}(\sigma)-s_{j}(\nu)$ in $\sigma$ rather than in $v$; the maximum allowable delay time of the original job $j$ in $v$ is defined as $\Delta_{j}=K-\left\{s_{j}(v)-r_{j}\right\}, j \in J_{o}$. Furthermore, consider a schedule $\eta$ ( $\eta$ can be an original schedule or a partial schedule including some rework jobs) such that if the relative sequence of the jobs in $\eta$ remains unchanged when the rest of the rework jobs are scheduled, then in $\eta$, the maximum allowable delay time associated with a job $[i]$ is $\Delta_{[i]}(\eta)=\min _{i \in J}\left\{\Delta_{[i]}, I_{[i+1]}+\Delta_{[i+1]}(\eta)\right\}$, where [i] indicates a job in the $i$ position and $I_{[i+1]}$ denotes the idle-time of the machine ahead of job $[i+1]$ immediately. Let $I=\sum I_{j}, j \in J_{O}$ denote the total idle-time ahead of original jobs in a schedule.

In this paper, rescheduling refers to the problem of updating $v$ to include unscheduled rework jobs available at time zero. Without loss of generality, it can be assumed that $r_{i}=0$ for $i \in J_{R}$ and that all $p_{j}$ and $r_{j}$ are known integers for $j \in J$. This assumption is justified when considering that rescheduling will typically occur periodically considering all known rework jobs available at that point of time.

\subsection{Problem formulation.}

In this paper, the objective of the rescheduling problem is to find a schedule $\sigma$ to minimize the maximum waiting time $w_{\max }(\sigma)$, where $w_{\max }(\sigma)=\max \left\{w_{i}(\sigma) \mid i \in J\right\}$. The Single-machine Rescheduling problem with Ready-times (SRR) is formulated in this paper as follows:

$$
\begin{aligned}
& (\mathrm{SRR}): \min w_{\max }(\sigma) \\
& \text { s. } t \text {. } \\
& w_{j}(\sigma) \leq K, \quad j \in J_{o} \\
& s_{j}(\sigma) \geq r_{j}, \quad j \in J \\
& s_{[j]}(\sigma)+p_{[j]} \leq s_{[j+1]}(\sigma), \quad j \in J \\
& s_{[i]}(\sigma)<s_{[j]}(\sigma), \quad s_{[i]}(\nu)<s_{[j]}(v),[i],[j] \in J_{O} \\
& \max \left\{p_{i} \mid i \in J_{R}\right\} \leq \min \left\{p_{j} \mid j \in J_{O}\right\}
\end{aligned}
$$

expression (1) is the objective function, expression (2) ensures that the waiting time of the original jobs is less than or equal to a given value, expression (3) ensures that a job is only scheduled after or at its ready-time, expression (4) ensures that only one job is processed at a time, expression (5) ensures that the relative sequence of the original jobs in $v$ remains unchanged after solving the rescheduling problem, and expression (6) ensures the maximum processing time of the rework jobs is smaller than the minimum processing time of the original jobs. Note that, (5) and (6) are technological constraints required to model practical conditions in the industry case mentioned earlier; namely, that the original sequence needs to remain unchanged, and that the rework jobs are shorter than a given threshold value s (i.e., we assume the threshold value is the maximum processing time).

In this paper, eight special cases of SRR are considered, among them seven cases can be solved 
optimally and one case can be solved 3/2-approximationoptimal. Table 1 provides a summary of our features, algorithms, computational complexity and results for eight special cases of the SRR problem studied.

\section{[Insert Table 1 Here]}

\subsection{Properties of the optimal solution (for SRR).}

In general, there may be some idle-times in front of the jobs in the original schedule because of the consideration of ready times. The following property formalizes the relation between idle-times and processing times in a feasible solution. According to the previous definition, $\eta$ is an active schedule that can be a schedule of original jobs or a partial schedule of original and rework jobs.

Property 1: A schedule $\eta^{\prime}$ obtains scheduling job $j$ immediately before job $[i]$ is feasible if $p_{j} \leq I_{[i]}+\Delta_{[i]}(\eta)$, where $[i] \in J,[i] \in \eta$ and $j \in J_{R}, j \notin \eta$.

Proof: If $p_{j} \leq I_{[i]}+\Delta_{[i]}(\eta)$, where $[i] \in J,[i] \in \eta$ and $j \in J_{R}, j \notin \eta$, i.e., in $\eta$, the total number of maximum allowable delay times and idle-times of the machine before job $[i],[i] \in J$ and $[i] \in \eta$ is not less than $p_{j}, j \in J_{R}$ and $j \notin \eta$. Consequently, inserting job $j$ into schedule $\omega$ immediately before job $i$ can satisfy the constraints of the model for SRR, the resulting schedule $\eta^{\prime}$ obtained is a feasible solution.

If a feasible schedule $\eta^{\prime}$ is obtained by inserting job $j$ into the schedule immediately before job $i$ in an active schedule $\eta$, then the constraints of the model for SRR should be satisfied. Thus, $w_{[i]}\left(\eta^{\prime}\right) \leq K$, and for every original job after job $j$, the waiting times are not longer than $K$. Therefore, the sum of the maximal delayable times and idle-times before the original job scheduled after job $j$ is not less than $p_{j}$ in $\eta$, i.e., $p_{j} \leq I_{[i]}+\Delta_{[i]}(\eta)$, where $[i] \in J,[i] \in \eta$ and $j \in J_{R}$, $j \notin \eta$.

The following property captures the condition when a pair-wise interchange between adjacent original and rework jobs results in an improved schedule.

Property 2: Given an original job $[i]$ followed by a rework job $j$ in a schedule $\sigma$, if $p_{j} \leq I_{[i]}+\Delta_{[i]}(\sigma)$, then exchanging job $i$ and $j$ results in a better schedule $\sigma^{\prime}$.

Proof: $p_{j} \leq I_{[i]}+\Delta_{[i]}(\sigma)$ can guarantee the feasibility of $\sigma^{\prime}$. According to constraint (6), $\max \left\{p_{i} \mid i \in J_{R}\right\} \leq \min \left\{p_{j} \mid j \in J_{O}\right\}$, a simple job interchanging argument can prove that $\sigma^{\prime}$ is better than or equal to $\sigma$. $\square$

For a given sequence of rework jobs, property 2 encourages scheduling the rework jobs as early as possible before the original jobs while satisfying constraint (2). This property is formalized in the following property.

Property 3: Given a sequence of rework jobs, an optimal solution can be obtained by inserting rework jobs following this sequence into the original schedule as early as possible while satisfying constraint (2). 
Property 4 defines a solution space that contains the optimal solution for SRR.

Property 4: Given a schedule of original jobs, the solution space consisting of inserting all permutations of the rework jobs, as early as possible, that satisfies constraint (2) contains the optimal solution to SRR.

The following property establishes an equivalent objective function for an optimal solution. This equivalency will be useful in the development of the solution procedure.

Property 5: An optimal solution to $\min \max \left\{w_{i}(\sigma) \mid i \in J_{R}\right\}$ subject to (2)-(6) is also optimal for SRR.

Proof: Let $\sigma^{\prime}$ be an optimal schedule for $\min \max \left\{w_{i}(\sigma) \mid i \in J_{R}\right\}$ subject to (2)-(5); we need to show that $w_{\text {max }}\left(\sigma^{\prime}\right)=\max \left\{w_{i}\left(\sigma^{\prime}\right) \mid i \in J\right\}=\max \left\{\max \left\{w_{i}\left(\sigma^{\prime}\right) \mid i \in J_{O}\right\}, \max \left\{w_{i}\left(\sigma^{\prime}\right) \mid i \in J_{R}\right\}\right\}$. Two cases will be considered:

Case 1: If $\max \left\{w_{i}\left(\sigma^{\prime}\right) \mid i \in J_{O}\right\} \leq \max \left\{w_{i}\left(\sigma^{\prime}\right) \mid i \in J_{R}\right\}$, then $w_{\max }\left(\sigma^{\prime}\right)=\max \left\{w_{i}\left(\sigma^{\prime}\right) \mid i \in J_{R}\right\}$ is optimal for SRR.

Case 2: If $\max \left\{w_{i}\left(\sigma^{\prime}\right) \mid i \in J_{O}\right\}>\max \left\{w_{i}\left(\sigma^{\prime}\right) \mid i \in J_{R}\right\}$, then $w_{\max }\left(\sigma^{\prime}\right)=\max \left\{w_{i}\left(\sigma^{\prime}\right) \mid i \in J_{O}\right\}$. We want to obtain a new schedule $\sigma^{\prime \prime}$, where $\max \left\{w_{i}\left(\sigma^{\prime \prime}\right) \mid i \in J_{O}\right\}<\max \left\{w_{i}\left(\sigma^{\prime}\right) \mid i \in J_{O}\right\}$ and at least a job $b$ before original job $d=\arg \max \left\{w_{i}\left(\sigma^{\prime}\right) \mid i \in J_{O}\right\}$ should be right-shifted after job $d$ in $\sigma^{\prime}$. The sequence of the original jobs cannot be changed due to constraint (5); thus, $b$ is a rework job, not an original job. Due to the ready-time constraints in (3), $w_{d}\left(\sigma^{\prime \prime}\right) \geq w_{d}\left(\sigma^{\prime}\right)-p_{b}$, $w_{b}\left(\sigma^{\prime \prime}\right) \geq w_{d}\left(\sigma^{\prime}\right)+p_{d}-p_{b}$. Because of constraint (6), $\max \left\{p_{i} \mid i \in J_{R}\right\} \leq \min \left\{p_{j} \mid j \in J_{o}\right\}$, $w_{b}\left(\sigma^{\prime \prime}\right) \geq w_{d}\left(\sigma^{\prime}\right) ;$ thus, $w_{\max }\left(\sigma^{\prime \prime}\right)=w_{b}\left(\sigma^{\prime \prime}\right) \geq w_{\max }\left(\sigma^{\prime}\right)$. $\square$

Moreover, because unscheduled rework jobs are available at time zero, the minimization of $\max \left\{w_{i}(\sigma) \mid i \in J_{R}\right\}$ is a regular performance measure. Inserting idle-times will not be beneficial for the objective function. Given that the sequence fully specifies the schedule, according to property 5 , the minimization of $w_{\max }(\sigma)$ is a regular performance measure and the following properties are duly obtained.

Property 6: For all optimal schedules of the SRR problem, in the final block of the rework jobs, the rework job of the longest processing time in this block is scheduled last.

Property 7: There exists an optimal schedule for SRR in which the rework jobs within each block of these rework jobs are sequenced in SPT order.

Properties 6 and 7 can be demonstrated using the pair-wise interchange argument.

\section{Complexity Analysis}

Theorem 1: The SRR problem is NP-hard.

Proof: Similar to Hall et al. (2004), this problem can be demonstrated to be NP-hard by reducing to the 3-Partition Problem of Garey et al. (1979).

3-Partition Problem: Given $3 t$ elements with integer sizes, where $\sum_{j=1}^{3 t} a_{j}=t \cdot b$ and $\frac{b}{4}<a_{j}<\frac{b}{2}$ for $j=1, \ldots, 3 t$, does there exist a partition $S_{1}, S_{2}, \ldots, S_{t}$ of the index set $\{1,2, \ldots, 3 t\}$ such 
that $\left|S_{1}\right|=3, \sum_{j \in S_{i}} a_{j}=b$ for $i=1, \ldots, t$.

Without loss of generality, suppose that there is a solution to the 3-Partition Problem, where the elements are well numbered such that $a_{3 l-2}+a_{3 l-1}+a_{3 l}=b$ for $l=1, \ldots, t$.

Consider the following problem instance of the SRR problem for objective function $w_{\max }(\sigma)<C$ : $n_{O}=9 t, n_{R}=3 t ; K=0, C=t-1+t b-\frac{b}{4} ;$

For original jobs: $p_{i}=1$ for $i=1, \ldots, 9 t$;

$$
r_{i}=\left\{\begin{array}{cc}
i b+i-1 & i=1, \ldots, t \\
t b+i-1 & i=t+1, \ldots, 9 t
\end{array} ;\right.
$$

For reworked original jobs:

$$
p_{i}=a_{j}, \frac{b}{4}<a_{j}<\frac{b}{2}, \sum a_{j}=t \cdot b, \text { for } i=9 t+1, \ldots 12 t, j=1, \ldots, 3 t .
$$

The item $C$ is a lower bound of the maximum waiting time of all jobs in $\sigma$. It should be proven that there exists a feasible schedule for this problem instance of the SRR problem for objective function $w_{\max }(\sigma)<C$ if and only if there exists a solution to the 3-Partition Problem (See Fig. 1).

\section{[Insert Fig. 1 Here]}

(Sufficiency) Consider a feasible solution $\sigma$ in which all original jobs have no waiting time. The start time of the original jobs is $s_{i}(\sigma)=r_{i}$ for $i=1, \ldots, 9 t$, where each original job is processed in a time interval of one unit, i.e., job 1 is processed in the $[\mathrm{b}, 1+b]$ time interval, job 2 is processed in $[1+2 b, 2+2 b], \quad \ldots$, job $t$ is processed in $[t-1+t b, t+t b]$, job $t+1$ is processed in $[t+t b, t+t b+1], \ldots$, job $9 t$ is processed in $[t b+9 t-1, t b+9 t]$. It is obvious that the above arrangement can lead to the optimum solution $v$ to the $1\left|r_{i}\right| w_{\max }(v), i \in J_{O}$ problem, where optimum value $w_{\max }(v)=0$. Because all original jobs have no waiting time in $\sigma$, the start time and the completion time of original jobs in $J_{O}$ in $\sigma$ remain unchanged as in the original schedule $v$, which gives rise to the machine having $t$ idle-time intervals. Therefore, it is possible to arrange the $3 t$ rework jobs in $\sigma$ by inserting the rework jobs $\{3 t+1, \ldots, 12 t\}$ into the $t$ waiting time intervals: $[0, b]$, $[1+b, 1+2 b], \ldots, \quad[t-1+t b-b, t-1+t b]$, such that 3 rework jobs are processed in each of the idle-time intervals (See Figure 1). Provided there exists a solution to the 3-Partition Problem, due to the waiting times of the original jobs being zero, the maximal waiting time of all jobs is the waiting time of the last rework job scheduled. This would be $w_{\max }(\sigma)=t-1+t b-p_{R}^{\text {last }}(\sigma)$, where $p_{R}^{\text {last }}(\sigma)$ is the processing time of the last rework job scheduled in $\sigma$. Because $\frac{b}{4}<p_{R}^{\text {last }}(\sigma)<\frac{b}{2}$, the schedule $\sigma$ makes $w_{\max }(\sigma)<C$ hold. The feasible rescheduled solution $\sigma$ would be obtained.

(Necessity) Consider a feasible schedule $\sigma$ with $w_{\text {max }}(\sigma)<C$. Because $w_{\text {max }}(v)=0$ and $K=0$, each original job in $\{1, \ldots, 9 t\}$ can start its processing as desired at time $r_{i}, i=1, \ldots, 9 t$ in the original optimal schedule $v$ and in the rescheduling solution $\sigma$. Additionally, the $3 t$ rework jobs are 
arranged in $t$ idle-times of the original schedule $v$. Because of $\frac{b}{4}<p_{R}^{\text {last }}(\sigma)<\frac{b}{2}$, the equality $w_{\max }(\sigma)=t-1+t b-p_{R}^{\text {last }}(\sigma)<C$ holds. Moreover, $\sum_{j=1+3 t}^{12 t} p_{j}=\sum_{j=1}^{3 t} a_{j}=t b$ ensures that the feasible schedule $\sigma$ has no idle-time and that all three of the rework jobs are processed in $b$ units of time, exactly the length of one of the $t$ idle-time intervals (i.e., $[0, b],[1+b, 1+2 b], \ldots$, $[t-1+t b-b, t-1+t b])$. This implies the existence of a solution to the 3-Partition Problem.

The reduction of the above proof shows that unless $\mathrm{P}=\mathrm{NP}$, the SRR problem has no M-approximation algorithm for every given $M>0$. To this end, one can construct an instance from the partition problem. The rework jobs correspond to the items in the partition instance. By setting $K=0$, by keeping two idle-time intervals (of equal length $b$ ) among the processing of the original jobs, and with only two original jobs of lengths 1 and $(2 b+1) M$ in this order, one can see that either partition instance has a solution and thus the maximum waiting time of the rework jobs is $2 b+1$. Otherwise, the partition instance has no solution and the maximum waiting time of the rework jobs is at least $(2 b+1)+(2 b+1) M+1>(2 b+1) M$. Still, a pseudo-polynomial algorithm and a heuristic algorithm are developed to solve some special cases.

\section{A Pseudo-Polynomial Algorithm for a Special Case}

In this section, a special case, which will be referred to as SRR-k, is studied. It satisfies $\left|s_{[j]}(v)+\Delta_{[j]}(\mathcal{U})>C_{[j-1]}(\mathcal{U})+\Delta_{[j-1]}(\mathcal{U})\right|=1, j=1, \ldots, n_{O}$. SRR-k means that only one idle time can be generated by delaying the starting times of all original jobs for their maximum allowable delay time in original schedule $v$. Two properties of the optimal solution are elaborated for the SRR-k case. The Knapsack-based Algorithm (KbA) is developed according to properties of the optimal solution for the SRR-k case as follows.

\subsection{The properties of the optimal solution for SRR-k.}

Property 8: In SRR-k, there exists an optimal schedule where the rework job with the maximum processing time is scheduled last for all rework jobs.

Proof: By a simple job interchanging argument. $\square$

Property 9: For the SRR-k problem, the objective of minimizing $w_{\max }(\sigma)$ is equivalent to minimizing $\max \left\{C_{i}(\sigma) \mid i \in J_{R} \backslash l\right\}$, where $l$ is a rework job with the maximum processing time.

Proof: Consider Property 5 and the following three cases:

Case 1: $\sum_{i \in J_{R} \backslash l} p_{i}>I$, i.e., some rework jobs in addition to $l$ are scheduled after the last original $\begin{array}{llllll}\text { job by } & \text { property } & 2 & \text { and }\end{array}$ $\max \left\{w_{i}(\sigma) \mid i \in J_{R}\right\}=\max \left\{C_{i}(\sigma) \mid i \in J_{R}\right\}-p_{l}=\max \left\{C_{i}(\sigma) \mid i \in J_{R} \backslash l\right\}$.

Case 2: $\sum_{i \in J_{R}} p_{i} \leq I$, i.e., the sum of processing times for all rework jobs is not more than the idle-time in SRR-k. The optimal schedule $\sigma^{*}$ can be obtained by scheduling all rework jobs in this idle-time and scheduling the rework job with maximum processing time to be last based on property 8 . Simultaneously, $\max \left\{C_{i}\left(\sigma^{*}\right) \mid i \in J_{R} \backslash l\right\}$ is optimal. 
Case 3: $\sum_{i \in J_{R} \backslash l} p_{i} \leq I$ and $\sum_{i \in J_{R}} p_{i}>I$. According to properties 2 and 8 , an optimal solution is obtained by scheduling the rework job $l$ after the last original job and scheduling the remaining rework jobs in the idle-time within $v^{\prime}$ for the SRR-k problem. $\square$

The following procedure, named $\mathrm{KbA}$, is derived using the above properties for SRR-k.

\section{2. $\mathrm{Kb} \boldsymbol{A}$ and its complexity}

Given properties 3, 8 and 9, the main idea of $\mathrm{KbA}$ is to delay the starting times of all original jobs for their maximum allowable delay time in original schedule $v$ first based on property 3 and, in turn, to insert the rework jobs (except the rework job with the maximum processing time) into this idle-time by means of a dynamic programming algorithm to minimize $\max \left\{C_{i}(\sigma) \mid i \in J_{R} \backslash l\right\}$ using property 9. Lastly, rework job $l$ with the maximum processing time is scheduled last using property 8 . The way rework jobs are inserted into the idle time is similar to Liu and Ro (2014) (i.e. case $\left.h_{1} \| S, k=+\infty, \mu=0\right)$; however, our algorithm maximizes the total processing time of rework jobs inserted, instead of minimizing a cost function of certain jobs. The detailed steps are as follows:

Step1. Update the starting times of original jobs to $s_{[j]}(v)+\Delta_{[j]}(v)$, and then calculate the idle-time $I=\max \left\{s_{[j]}(v)+\Delta_{[j]}(v)-C_{[j-1]}(v)+\Delta_{[j-1]}(v)\right\}$, where $j=1, \ldots, n_{O}$.

Step2. Find the rework job $l$ with the maximal processing time within $J_{R}$.

Step 3. The set of candidate rework jobs is $J_{c}=J_{R} \backslash l$. A dynamic programming algorithm is devised to solve knapsack problems that have $n_{R}-1$ rework jobs in $J_{c}$ and an idle-time $I$. The processing time of rework job $i$ is $p_{i}$.

State variable: A set of candidate rework jobs $J_{c}$ and the remainder idle-time $I_{r}$.

Decision variable: The rework job $i$ is either inserted into the idle-time or not.

State transition: $\left\{\begin{array}{l}J_{c}=J_{c} \backslash i, I_{r}=I_{r}-p_{i}, \text { for } I_{r} \geq p_{i} \\ J_{c}=J_{c}, I_{r}=I_{r}, \text { for } I_{r}<p_{i}\end{array}\right.$.

Value function: The value obtained $V$ equal to the sum of the value for the first $i$ rework jobs inserted into the idle-time $I_{r}$ for $i \in J_{c}, 0 \leq I_{r} \leq I$.

Recurrence equation: $\left\{\begin{array}{l}V\left(i, I_{r}\right)=\max \left\{V\left(i, I_{r}\right), V\left(i-1, I_{r}-p_{i}\right)\right\}, I_{r} \geq p_{i} \\ V\left(i, I_{r}\right)=V\left(i-1, I_{r}\right), I_{r}<p_{i}\end{array}\right.$.

Step 4. If $J_{c} \neq \varnothing$, schedule the rework jobs in the set of remainder rework jobs $J_{c}$ after the last original job in $v$ randomly. Otherwise, go to step 5.

Step 5. Schedule the rework job $l$. If remainder idle-time $I_{r}$ is more than or equal to $p_{l}$, the rework job $l$ is scheduled after the last rework job. Otherwise, the rework job $l$ is scheduled last.

The time complexity of Step 1 is $O\left(2 n_{O}\right)$, Step 2 is $O\left(n_{R}\right)$, and Step 3 is $O\left(n_{R} I\right)$, and the sum of Step 4 and Step 5 is $O\left(n_{R}\right)$; thus, the time complexity of $\mathrm{KbA}$ is $O\left(2 n_{O}+2 n_{R}+n_{R} I\right)$.

\subsection{Analysis of $\mathrm{Kb} A$.}

$\mathrm{KbA}$ is a pseudo-polynomial algorithm that was mentioned by Martello et al. (1997) in fact 
because computing time depends on the size of the idle-time $I$. We then have the following theorem.

Theorem 2: KbA finds an optimal schedule $\sigma^{*}$ in pseudo-polynomial time for the SRR problem with $\left|s_{[j]}(v)+\Delta_{[j]}(v)>C_{[j-1]}(v)+\Delta_{[j-1]}(v)\right|=1, j=1, \ldots, n_{O}$.

Proof: KbA delays the starting times of all original jobs for their maximum allowable delay time in original schedule $v$ first based on property 2 . The algorithm schedules rework jobs as early as possible as long as constraint 2 is satisfied. Step 4 obtains the optimal solution to minimizing $\max \left\{C_{i}(\sigma) \mid i \in J_{R} \backslash l\right\}$ in SRR-k. Thus, step 5 schedules the job $l$ last, solving the SRR-k problem (Properties 8 and 9). $\square$

\section{A Heuristic for General SRR}

A heuristic named Shortest processing time Insert Heuristic (SIH) is developed based on the properties developed in section 2 to solve the general SRR. The optimality of SIH for several special cases is also discussed.

\subsection{SIH and its complexity}

Given properties 6 and 7, the basic idea behind the SIH algorithm is as follows: the rework jobs are sorted by SPT rule and are inserted into the original schedule as early as possible in terms of jobs group (properties 1 and 2) such that $\max \left\{w_{i}(\sigma) \mid i \in J_{R}\right\}$ is minimized using property 5. Fig. 2 shows the flow chart of SIH. The detailed steps for SIH are as follows:

\section{[Insert Fig. 2 Here]}

Step 1. Input the rework jobs in non-descending order, i.e., $\omega=\left\{n_{O}+1, \ldots, n_{O}+n_{R}\right\}$. Let $P_{l}=\sum_{j=1}^{l} p_{j}, \quad l \in\{1, \ldots,|\omega|\}$ indicate the sum of processing times for the rework jobs in the first $l$ positions in $\omega$.

Step 2. Let $\sigma=\varnothing, \tilde{I}=I_{[1]}$, where $I_{[1]}$ denotes the idle-time ahead of the first original job in original schedule $v$.

Step 2.1. If $v \neq \varnothing, \omega \neq \varnothing$, go to Step 2.1.1. If $v \neq \varnothing, \omega=\varnothing$, then update $\sigma$ to $\sigma$ followed by $v$, i.e., $\sigma=\sigma \mid v$. If $v=\varnothing, \omega \neq \varnothing$, then $\sigma=\sigma \mid \omega$.

Step 2.1.1. In $v$, let $i=2$. If $C_{[i-1]}(v)<s_{[i]}(v)$ and $s_{[i]}(v)=r_{[i]}$, then calculate $\Delta_{\min }=\min _{a=1, \ldots, i-1}\left\{\Delta_{[a]}(v)\right\}$ in the first $i$ positions and $\Delta=\min \left\{\Delta_{\min }, r_{[i]}-C_{[i-1]}(v)\right\}$. Find the original job $[m]$, where $m=\underset{a=1, \ldots, i-1}{\arg \min }\left\{\Delta_{[a]}(v)\right\}$, and let $\tilde{J}^{o}=\{[j] \mid j \leq m,[j] \in v\}$ be the set of the original job from the first position original job to the first $m$ position original job in $v$. Go to Step 2.2. Otherwise, $i=i+1$, if $i>|v|$, and $\Delta=\min _{a=1, \ldots, n_{O}}\left\{\Delta_{[a]}(v)\right\}$. Find the original job $[m]$, where $m=\underset{a=1, \ldots, n_{O}}{\arg \min }\left\{\Delta_{[a]}(v)\right\}$.

Let $\tilde{J}^{o}=\{[j] \mid j \leq m,[j] \in v\}$ and go to Step 2.2. Otherwise, go to Step 2.1.1.

Step 2.2. If $\exists \quad P_{l} \leq \Delta+\tilde{I}$ and $P_{l+1}>\Delta+\tilde{I}$, where $P_{|\omega|+1}=+\infty$, then select 
$\tilde{J}^{R}=\left\{n_{O}+1, \ldots, n_{O}+l\right\}$ in $\omega$, let $\omega=\omega \backslash \tilde{J}^{R}$, and go to Step 2.2.1. Otherwise, $\tilde{J}^{R}=\varnothing, \omega=\omega$. If $\Delta=r_{[i]}-C_{[i-1]}(v)$, then $\sigma=\sigma$ and $\tilde{I}=\Delta+\tilde{I}$. Update all start times of jobs in $v$. Let $s_{[1]}(v)=s_{[1]}(v)+\Delta$; if $\Delta=\Delta_{\min }$, then $\sigma=\sigma \mid \tilde{J}^{o}, v=v \backslash \tilde{J}^{o}, \tilde{I}=r_{[i]}-C_{[i-1]}(v)$. Update all start time of jobs in $v$, and let $s_{[1]}(v)=s_{[1]}(v)+\Delta$. Go to Step 2.1.

Step 2.2.1. If $\Delta=r_{[i]}-C_{[i-1]}(v)$, then $\tilde{I}=\Delta+\tilde{I}-P_{l}, \sigma=\sigma \mid \tilde{J}^{R}$. Update all start times of jobs in $v$, and let $s_{[1]}(v)=s_{[1]}(v)+\Delta$; if $\Delta=\Delta_{\min }$, then $\sigma=\sigma\left|\tilde{J}^{R}\right| \tilde{J}^{o}, v=v \backslash \tilde{J}^{o}, \tilde{I}=\Delta+\tilde{I}-P_{l}$. Update all start times of jobs in $v$. Let $s_{[1]}(v)=s_{[1]}(v)+\Delta$. Go to Step 2.1.

The time complexity of step 1 is $O\left(n_{R} \log n_{R}\right)$, of step 2 is $O\left(n_{O}\right)$, of step 2.1 is $O\left(n_{O}\right)$, and of step 2.2 is $O\left(n_{R}\left(n_{R}+2 n\right)\right)$; thus, the time complexity of SIH is $O\left(n_{O}{ }^{2}+n_{O} n_{R}^{2}+2 n n_{O} n_{R}\right)$.

\subsection{Analysis of SIH.}

Six cases for which SIH is optimal and one case where SIH is a 3/2-approximation algorithm are discussed in this section.

Because of ready-time, the solutions of SIH for the SRR problem are, in general, schedules with idle-times between blocks of contiguous jobs. Given that the ready time for rework jobs is zero, there will be no idle-time in front of rework jobs in an optimal solution. The following theorem elaborates the optimality of SIH for the first case: relations between idle-times and an optimal solution of SRR.

Theorem 3: For general SRR, if $\sigma$ obtained by SIH does not have idle-time before the latest rework job, then $\sigma$ is optimal.

Proof: By a simple job interchanging argument. $\square$

Consider the second special case without idle-times in the original schedule (the original schedule constitutes a single block of jobs) for SRR, i.e., $s_{[j]}(v)=C_{[j-1]}(v), j=1, \ldots, n_{O}$. This special case is denoted as SSR-b.

Because there is no idle-time on the machine in the original schedule and the ready time of rework jobs is zero, it is easy to prove that the optimal schedule for the SSR-b problem does not have idle-times. The following property is useful for proving the optimality of SIH for SSR-b.

Property 10: There exists an optimal schedule for SSR with $s_{[j]}(v)=C_{[j-1]}(v), j=1, \ldots, n_{O}$ in which the rework jobs are sequenced in SPT order.

Proof: By a simple job interchanging argument. $\square$

The following corollary proves the optimality of SIH using property 10 and theorem 3.

Corollary 1: SIH finds an optimal schedule $\sigma^{*}$ for SRR with $s_{[j]}(v)=C_{[j-1]}(v), j=1, \ldots, n_{O}$ in polynomial time.

Proof: It is proven by property 10 and theorem 3 .

Fig. 3 shows a problem instance concerning corollary 1 .

For original jobs, $p_{1}=12, p_{2}=10, p_{3}=10, p_{4}=8, p_{5}=13$;

$$
r_{1}=0, r_{2}=12, r_{3}=20, r_{4}=27, r_{5}=38 ;
$$

For rework jobs, $p_{6}=1, p_{7}=2, p_{8}=3$; 


$$
K=9 ; \quad w_{\max }\left(\sigma^{*}\right)=w_{8}=44
$$

\section{[Insert Fig. 3 Here]}

The third special case of SRR requires that the maximum allowable delay time of each original job in $v$ is not shorter than the maximum processing time of all rework jobs, i.e., $\Delta_{j}=K-\left\{s_{j}(v)-r_{j}\right\} \geq \max \left\{p_{i} \mid i \in J_{R}\right\}$ for $j \in J_{O}$.

Corollary 2: SIH can find an optimal schedule $\sigma^{*}$ for the SRR problem in polynomial time if $\Delta_{j} \geq \max \left\{p_{i} \mid i \in J_{R}\right\}$ for $j \in J_{O}$.

Proof: $\Delta_{j}(v) \geq \max \left\{p_{i} \mid i \in J_{R}\right\}$ for $j \in J_{O}$ regardless of whether there are idle times in the original schedule $v$. In $v$ or a partial schedule (i.e., some rework jobs have been inserted into $v$ ), if there is no idle-time ahead of an original job, there is no idle time ahead of this original job even if rework jobs are inserted before this original job. If there is an idle-time ahead of an original job, one or more rework jobs can be scheduled ahead of this original job because the maximum allowable delay time of this original job in $v$ or in the partial schedule is not shorter than the maximum processing time of all rework jobs. There is no idle time ahead of the rework job scheduled last. According to theorem 3, SIH can find an optimal schedule $\sigma^{*}$ for the SRR problem in polynomial time if $\Delta_{j} \geq \max \left\{p_{i} \mid i \in J_{R}\right\}$ for $j \in J_{O}$.

Fig. 4 shows a problem instance concerning corollary 2.

For original jobs, $p_{1}=10, p_{2}=10, p_{3}=12, p_{4}=8, p_{5}=13, p_{6}=8, p_{7}=6$;

$$
r_{1}=0, r_{2}=6, r_{3}=21, r_{4}=29, r_{5}=42, r_{6}=56, r_{7}=73 \text {; }
$$

For rework jobs, $p_{8}=2, p_{9}=2, p_{10}=3, p_{11}=4$;

$$
K=8 ; \quad w_{\max }\left(\sigma^{*}\right)=w_{11}=60 .
$$

\section{[Insert Fig. 4 Here]}

The fourth special case of SRR, in which all rework jobs have a unified processing time, i.e., the SRR- $p$ case, is considered as follows, i.e., $p_{j}=p, j \in J_{O}$, where $p$ is a constant.

Proposition 1: $\mathrm{SIH}$ can find an optimal schedule $\sigma^{*}$ for the $\mathrm{SRR}$ with $p_{j}=p, j \in J_{O}$ in polynomial time.

Proof: The SRR- $p$ case means that all of the rework jobs have the same processing time. Thus, SIH reschedules the rework jobs as early as possible to obtain the optimal solution for SRR- $p$ based on properties 2 and 5.

Fig. 5 shows a problem instance illustrating proposition 1.

For original jobs, $p_{1}=10, p_{2}=10, p_{3}=10, p_{4}=8, p_{5}=13$;

$$
r_{1}=1, r_{2}=3, r_{3}=24, r_{4}=26, r_{5}=43 \text {; }
$$

For rework jobs, $p_{6}=p_{7}=p_{8}=2$; 


$$
K=8 ; w_{\max }\left(\sigma^{*}\right)=w_{8}=44 .
$$

\section{[Insert Fig. 5 Here]}

Three special cases regarding the relation between rework jobs and the original schedule are discussed for SRR.

In the fifth special case of SRR, all rework jobs can be scheduled ahead of the first original job, i.e., $\quad I_{[1]}+\Delta_{[1]}(v) \geq \sum_{i \in J^{R}} p_{i}$.

Proposition 2: SIH can find an optimal schedule $\sigma^{*}$ for the SRR problem with $I_{[1]}+\Delta_{[1]}(\nu) \geq \sum_{i \in J^{R}} p_{i}$ in polynomial time.

Proof: Given SIH, all rework jobs are sequenced in SPT order before the first original job and scheduling $\sigma$ is obtained, where $\max \left\{w_{i}(\sigma) \mid i \in J_{R}\right\}=\sum_{i \in J^{R}} p_{i}-\max \left\{p_{i} \mid i \in J_{R}\right\}$. This theorem is proven by a simple job interchanging argument.

Fig. 6 shows a problem instance corresponding to proposition 2.

For original jobs, $p_{1}=10, p_{2}=10, p_{3}=12, p_{4}=13, p_{5}=13, p_{6}=8$;

$$
r_{1}=0, r_{2}=8, r_{3}=25, r_{4}=36, r_{5}=47, r_{6}=65 \text {; }
$$

For rework jobs, $p_{7}=1, p_{8}=1, p_{9}=3$;

$$
K=8 ; w_{\max }\left(\sigma^{*}\right)=w_{2}=7 \text {. }
$$

\section{[Insert Fig. 6 Here]}

Consider the sixth special case of SRR, where no rework job can be scheduled into the original schedule. All rework jobs can only be scheduled behind the original job in the last position in the original schedule, i.e., $I_{[j]}+\Delta_{[j]}(v)<\min \left\{p_{i} \mid i \in J_{R}\right\}$ for $[j] \in J_{O}$.

Proposition 3: SIH can find an optimal schedule $\sigma^{*}$ for the SRR problem with $I_{[j]}+\Delta_{[j]}(v)<\min \left\{p_{i} \mid i \in J_{R}\right\}$ for $[j] \in J_{O}$ in polynomial time.

Proof: $I_{[j]}+\Delta_{[j]}(v)<\min \left\{p_{i} \mid i \in J_{R}\right\}$ for $[j] \in J_{O}$ means that there is no rework job that can be settled ahead of the last original job. It is proven by a simple job interchange argument. $\square$

Fig. 7 shows a problem instance illustrating proposition 3.

For original jobs, $p_{1}=10, p_{2}=10, p_{3}=12, p_{4}=13, p_{5}=10$;

$$
r_{1}=0, r_{2}=4, r_{3}=21, r_{4}=27, r_{5}=48 \text {; }
$$

For rework jobs, $p_{6}=3, p_{7}=3, p_{8}=5$;

$$
K=8 ; w_{\text {max }}\left(\sigma^{*}\right)=w_{8}=62 \text {. }
$$


Now, we consider the seventh case, in which the sum of idle-times is less than the sum of processing times of all rework jobs, where the idle-times in front of the original jobs are obtained by delaying the start-times of original jobs as much as possible in the original schedule $v$, i.e., $I(v)+\Delta_{\left[n_{0}\right]}<\sum_{i \in J_{R}} p_{i}$

Theorem 4: SIH is a 3/2-approximation algorithm for the SRR problem with $I(v)+\Delta_{\left[n_{0}\right]}<\sum_{i \in J_{R}} p_{i}$ in polynomial time.

Proof: $I(v)+\Delta_{\left[n_{O}\right]}$ is equal to the longest available time of the machine before the job $\left[n_{O}\right]$, irrespective of whether the original jobs before the job $\left[n_{0}\right]$ are right-shifted. The SRR problem with $I(v)+\Delta_{\left[n_{0}\right]}<\sum_{i \in J_{R}} p_{i}$ has at least one rework job scheduled after the last original job in its optimal solution $O P T$.

Schedule $\sigma$, obtained by the SIH algorithm, can be derived when the idle-times are preceded by one of the original jobs and any idle-time $I_{j}$ ahead of the original job $j$ is less than $\max \left\{p_{i} \mid i \in J_{R}\right\}$. Because there are $n_{O}$ original jobs, there are at most $n_{O}$ idle-times and $I(\sigma) \leq n_{O} \cdot \max \left\{p_{i} \mid i \in J_{R}\right\}$. With the constrain (6) of $\max \left\{p_{i} \mid i \in J_{R}\right\} \leq \min \left\{p_{j} \mid j \in J_{O}\right\}$,

$$
\begin{aligned}
& n_{O} \cdot \max \left\{p_{i} \mid i \in J_{R}\right\} \leq \sum_{i=1}^{n_{O}} p_{i}, \text { such that } I(\sigma) \leq \sum_{i=1}^{n_{O}} p_{i}, \\
& \text { and } 2 I(\sigma) \leq I(\sigma)+\sum_{i=1}^{n_{O}} p_{i} \leq I(v)+\sum_{i=1}^{n_{O}} p_{i}=\max \left\{C_{i}(v) \mid i \in J_{O}\right\} \leq O P T
\end{aligned}
$$

Thus $I(\sigma) \leq \frac{1}{2} O P T$. By $\max \left\{C_{i}(\sigma) \mid i \in J_{R}\right\}>\max \left\{C_{i}(\sigma) \mid i \in J_{O}\right\}$ and Property $5, w_{\max }(\sigma)$ can be obtained:

$$
\begin{aligned}
w_{\max }(\sigma) & =\max \left\{w_{i}(\sigma) \mid i \in J_{R}\right\}=\max \left\{C_{i}(\sigma) \mid i \in J_{R}\right\}-\max \left\{p_{i} \mid i \in J_{R}\right\} \\
& =\sum_{i=1}^{n_{O}} p_{i}+\sum_{j=n_{O}+1}^{n_{O}+n_{R}} p_{j}+I(v)-(I(v)-I(\sigma))-\max \left\{p_{i} \mid i \in J_{R}\right\} \\
& =\sum_{i=1}^{n_{O}} p_{i}+\sum_{j=n_{O}+1}^{n_{O}+n_{R}} p_{j}-\max \left\{p_{i} \mid i \in J_{R}\right\}+I(\sigma)
\end{aligned}
$$

Since $\sum_{i=1}^{n_{O}} p_{i}+\sum_{j=n_{O}+1}^{n_{O}+n_{R}} p_{j}-\max \left\{p_{i} \mid i \in J_{R}\right\} \leq O P T$, then, $w_{\max }(\sigma) \leq \frac{3}{2} O P T . \square$

\section{Experimental Study}

In this section, we give some numerical results to evaluate the performance of the proposed SIH. A Branch-and-Bound algorithm (BB) was developed to obtain the optimal solution for the test problem. The BB is described in the appendix. The SIH and BB algorithms were programmed and tested using a C++ compiler on a machine with 8GB RAM, Intel Corei7-2600 CPU with 3.4 GHZ.

Five factors are considered in the generation of random problem instances. Table 2 summarizes these factors, their descriptions and their levels. 


\section{[Insert Table 2 Here]}

We first select $n$ equal to 20 or 40 and $n_{O} \in\{0.25 n, 0.5 n, 0.75 n\}$. The processing times of rework jobs are generated between 1 and $m$ and the processing times of original jobs are generated between $m$ and 100, randomly, where $m \in\{20,40\}$. Assuming that the original jobs are indexed so that $J_{O}=\left\{1, \ldots, n_{O}\right\}$, we generate an original schedule by sequencing these jobs in the order $\left(1, \ldots, n_{O}\right)$ and then insert idle time periods. The lengths of the idle time periods follow the same distribution as the processing times of the jobs. Let FIT denote the frequency of idle time within the original schedule; for example, if FIT $=0.1$ and $n_{O}=20$, then the expected number of idle time periods is $0.1 \times 20=2$. FIT $\in\{0.1,0.3,0.5,0.7\}$ are considered, respectively. The release times of the original jobs are considered one by one. The release time of the $j$ th original job is determined as follows: Current Available time of Machine (CAM) is a variable, and CAM equals the completing time of the original job preceding the $j$ th original job, with its initial value equal to zero. If the $j$ th original job proceeds an idle time, then its release time equals to the sum of CAM and the idle time. Otherwise, its release time equals an integer value between CAM and CAM $(j+1) \div(j+3)$ randomly. Repeat this process until the release time of the last original job is determined. Then, an original schedule is obtained. The value of $K$ is determined by the greater of the maximum waiting times of the original jobs in the original schedule and a parameter $\mu \in\{0.1,0.3,0.5,0.7\}$ multiplied by the expected sum of the completing times of the original jobs in the original schedule, in which $\mu$ models a wide range of tightness for the maximum waiting time constraint. The expected the sum of the completing times of the original jobs is obtained by multiplying the average number of the maximum and minimum completing time by the number of original jobs, i.e.

$$
K=\max \left\{\max \left(w_{i}(v) \mid i \in J_{O}\right), \mu \times n_{O} \times\left(\max \left(C_{i}(v) \mid i \in J_{O}\right)+\min \left(C_{i}(v) \mid i \in J_{O}\right)\right) \div 2\right\} .
$$

The number of combinations of different problem types tested is $2 \times 3 \times 2 \times 4 \times 4=192$. We randomly generate 10 problem instances for each of the 192 possible combinations, resulting in 1,920 problem instances.

The optimal solutions were obtained using BB. We set the running time limit to 90,000 seconds per problem instance. The solutions of 1,438 problems were obtained within the time limit. All of instances with $n=20$ were solved, while only $49.8 \%$ of the problems with $n=40$ were solved within the pre-specified time limit.

The running time performance of the BB algorithm is significantly affected by the number of rework jobs. Conversely, the running time performance of $\mathrm{BB}$ is not significantly influenced by the size of the processing time of rework jobs, frequency of idle time within the original schedule, and the degree of tightness for the maximum waiting time constraint.

The 1,438 optimal solutions of instances are used to test the performance of SIH. NO and CNO denote the number of instances for which an optimal solution is obtained by BB and SIH, respectively, and RCNO represents the CNO-to-NO rate. Let $z_{i}^{S I H}$ and $z_{i}^{B B}$ indicate the solution obtained by SIH 
and $\mathrm{BB}$, respectively. Relative error is $E_{i}=\left(z_{i}^{S H}-z_{i}^{B B}\right) / z_{i}^{B B}$, where $i=1, \ldots, N$. Average relative error is $A E=\sum_{i=1}^{N} E_{i} / N$. The standard deviation of $E_{i}$ is $S D=\sqrt{1 / N \sum_{i=1}^{N}\left(E_{i}-\bar{E}\right)^{2}}$, where $\bar{E}=\sum_{i=1}^{N} E_{i} / N . N$ is the number of instances within the candidate instant set.

The computation time for SIH is approximately 2 seconds, even when the number of jobs is 1000 . SIH obtains the optimal solution in 1,364 of the 1,438 cases where the optimal solution is known. Furthermore, $A E$ is 0.000871 and $S D$ is 0.013056 . The effect of each factor on the performance of SIH is presented in Tables 3 to 7 :

\section{[Insert Table 3 Here]}

\section{[Insert Table 4 Here]}

The results in Tables 3 and 4 suggest that SIH performs better as the problem size and processing time of a rework job increase.

\section{[Insert Table 5 Here]}

The results in Table 5 suggest that RCNO decreases as the percent of rework jobs increases. The performance of SIH is reduced with increasing percentage of rework jobs in the total jobs because the number of rework jobs increases.

\section{[Insert Table 6 Here]}

The results in Tables 6 suggest that the frequency of idle time within the original schedule does not significantly affect the performance of SIH.

\section{[Insert Table 7 Here]}

The results in Table 7 suggest that, except for the case where the maximum waiting time constraint is very tight (i.e., $\mu=0.1$ ), SIH finds the optimal solution in most cases; only one instance cannot be solved optimally on $\mu$ of both 0.3 and 0.7. A larger $K$ is associated with a larger $\mu$, resulting in more rework jobs scheduled ahead of the original job due to large shifts allowed by the original jobs in the original schedule, thus satisfying theorem 3 in most cases.

\section{Conclusions}

This paper studies a challenging rescheduling problem that is of particular importance for the quartz manufacturing industry. The theoretical importance of the paper resides in the formal complexity analysis of the problem work, the formalization of several structural properties of the 
problem, the development of effective solution procedures, and the proofs of the optimal or 3/2-approximation algorithms for eight special cases of SRR. The numerical experiments suggest that the heuristic yields close to optimal solutions that can solve problems similar to those found in practice.

Future work may investigate the case where the new (rework) jobs also have a ready-time. A more practical extension could entail solving the flow-shop and job-shop versions of the problem.

\section{Appendix}

\section{A Branch-and-Bound (BB) algorithm for the general SRR problem}

The following is the scheme for the proposed $\mathrm{BB}$ algorithm: during the computation, we keep a list of unexplored nodes arranged in increasing order according to the lower bounds of nodes, with ties broken by a non-increasing number of scheduled rework jobs. Each node represents a partial schedule. The root node is the original schedule. The algorithm always tries to develop the orders and the inserted positions for rework jobs. Before any new node is created, a series of dominance properties are checked. For each node of the search tree, which cannot be eliminated by dominance properties, a lower bound and an upper bound are calculated. If the lower bound is greater than or equal to the maximum waiting time of a known complete solution, this node is also eliminated. If the lower bound equals to the upper bound, then an optimal solution is obtained.

\section{Upper bounds}

The heuristic algorithm SIH is used to obtain upper bounds in every node. According to theorem 3, if the machine has no idle time in the initial rescheduling solution, then an optimal reschedule $\sigma^{*}$ has been obtained.

\section{Lower bounds}

Deleting all idle times of the original schedule equals to relaxing constraint condition (3) in the model of the SRR problem. According to theorem 3, SIH can find an optimal schedule for this relaxed SRR problem, and this optimal solution is a lower bound of the SRR problem.

\section{BB strategy}

In this algorithm, an original schedule is produced using a method similar to Hall's (2004). According to properties 3 and 4, the orders in full permutation for rework jobs are developed, a new rework job is inserted after the last rework job scheduled before the original jobs as early as possible in the partial schedule of current nodes, and then a new supplementary node is generated. Supplementary nodes are eliminated by using properties 6 and 7. The dominance properties used are properties 1 and 2 , as well as the dominance of the active subset.

The BB algorithm is explained by Case I as follows. Table 8 includes information pertaining to the original jobs and rework jobs. Figure 8 is the detailed branch-and-bound for Case I .

Case I . $J_{O}=\{1, \ldots, 8\}, J_{R}=\{9, \ldots, 12\}, K=20, v=\{1,2,3,4,5,6,7,8\}$.

\section{[Insert Table 8 Here]}

\section{[Insert Fig. 8 Here]}




\section{References}

Garey, M. R., \& Johnson, D. S. (1979).Computers and Intractability: A Guide to the Theory of NP-Completeness. Freeman, San Francisco, CA.

Hall, N. G., Liu, Z., \& Potts, C. N. (2007). Rescheduling for multiple new orders. INFORMS Journal on Computing, 19, 633-645.

Hall, N. G., \& Potts, C. N. (2004). Rescheduling for new orders. Operations Research. 52, 440-453.

Herrmann, J.W. (2006). Rescheduling strategies, policies, and methods. Handbook of Production Scheduling, 89, 135-148.

Hoogeveen, H., Lenté, C., \& V. T'kindt. (2012). Rescheduling for new orders on a single machine with setup times. European Journal of Operational Research, 223, 40-46.

Leon, V.J. (1991). Game-theoretic control and robust scheduling of job-shops in the presence of disruptions. Unpublished dissertation, Lehigh University, Bethlehem, Pennsylvania, May 1991.

Liu, Z., Y.K. Ro. (2014). Rescheduling for machine disruption to minimize makespan and maximum lateness. Journal of Scheduling, 17, 339-352.

Martello, S., Pisinger, D., \& Toth, P. (2000). New trends in exact algorithms for the 0-1 knapsack problem. European Journal of Operational Research, 123, 325-332.

Unal, A. T., Uzsoy, R., \& Kiran, A. S. (1997). Rescheduling on a single machine with part-type depend setup times and deadlines. Annals of Operations Research, 70, 93-113.

Vieira, G., Herrmann, \& J., Lin, E. (2003). Rescheduling manufacturing systems: a framework of strategies, policies, and methods. Journal of Scheduling, 6, 39-62.

Wu, S. D., Storer, R. H., \& Chang, P. C. (1992). A rescheduling procedure for manufacturing systems under random disruptions, in: G. Fandel, T.Gulledge, A. Jone (Eds.), New Directions for Operations Research in Manufacturing, Springer, Berlin, Germany, 292-308.

Yang, B. (2007). Single machine rescheduling with new jobs arrivals and processing time compression. International Journal of Advanced Manufacturing Technologies, 34, 378-384.

Yuan, J., \& Mu, Y. (2007). Rescheduling with release dates to minimize makespan under a limit on the maximum sequence disruption. European Journal of Operational Research, 182, 936-944.

Zhao, C., \& Tang, H. (2010). Rescheduling problems with deteriorating jobs under disruptions. Applied Mathematical Modeling, 34, 238-243. 


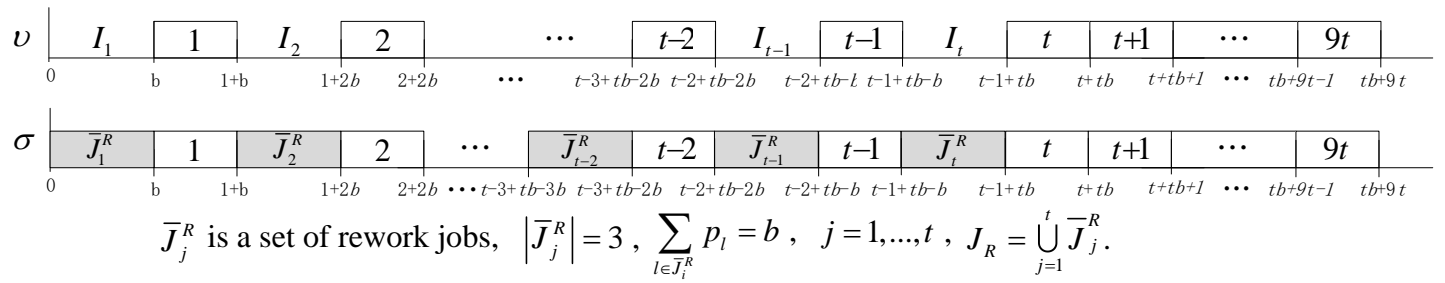

Fig.1. Illustration of SRRJ vs. 3-Partition

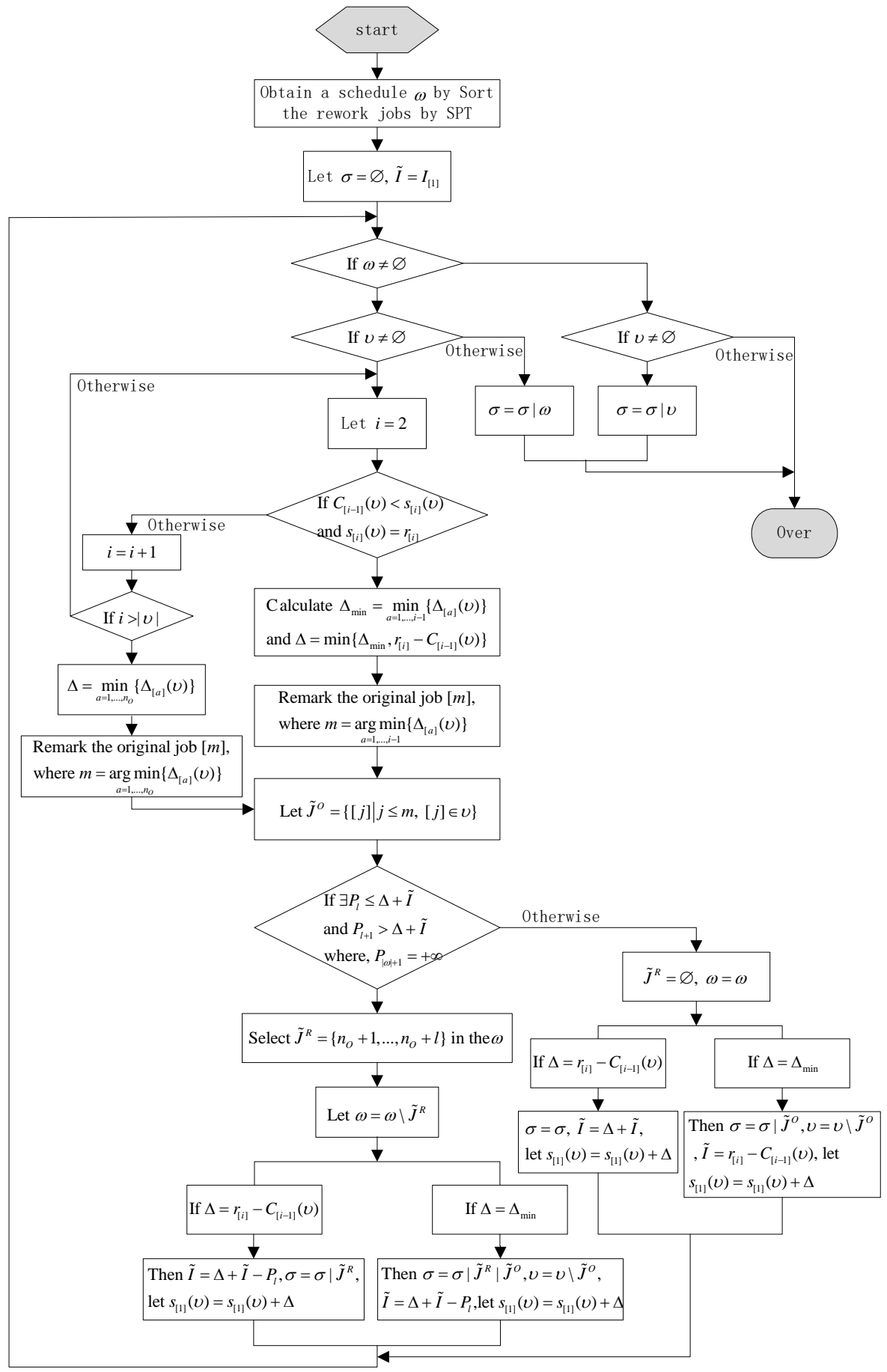

Fig.2. The flow chart of SIH 


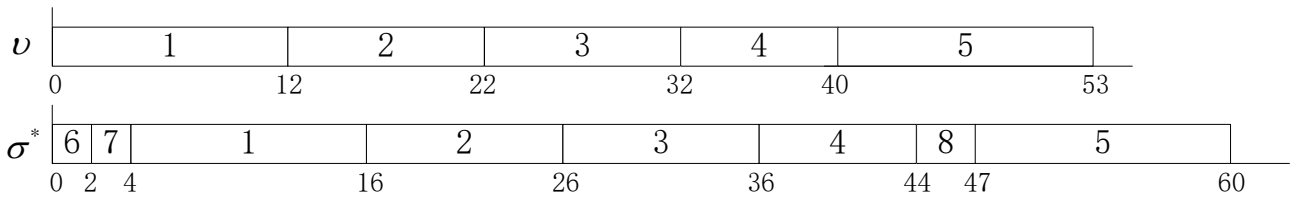

Fig.3. Case of corollary 1

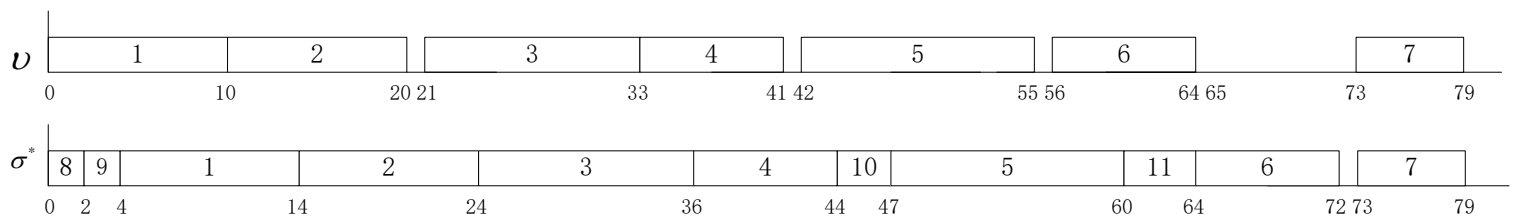

Fig.4. Case of corollary 2

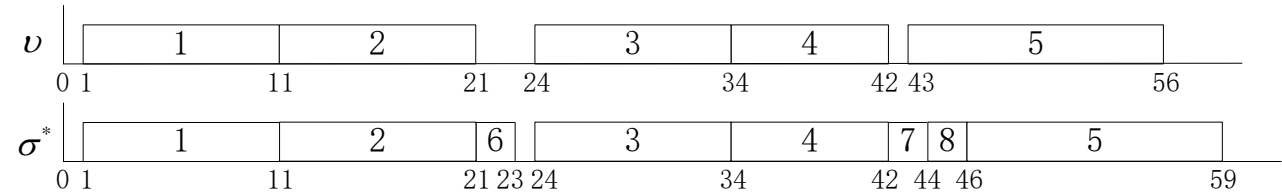

Fig.5. Case of proposition 1

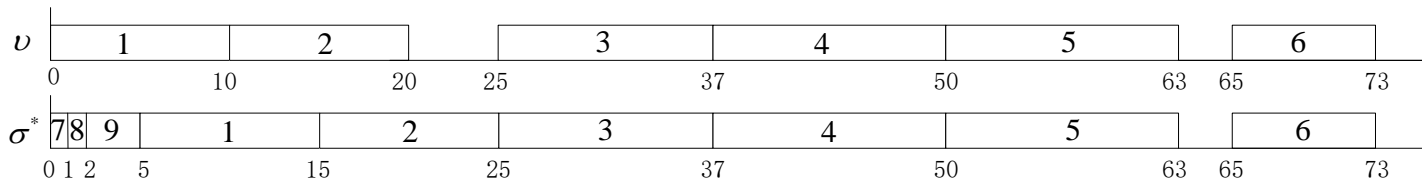

Fig.6. Case of proposition 2

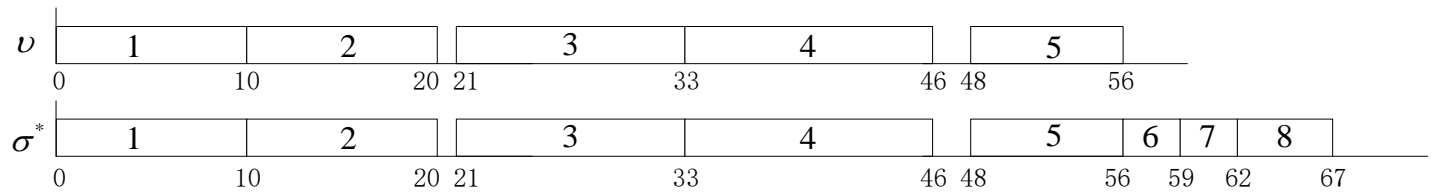

Fig.7. Case of proposition 3 

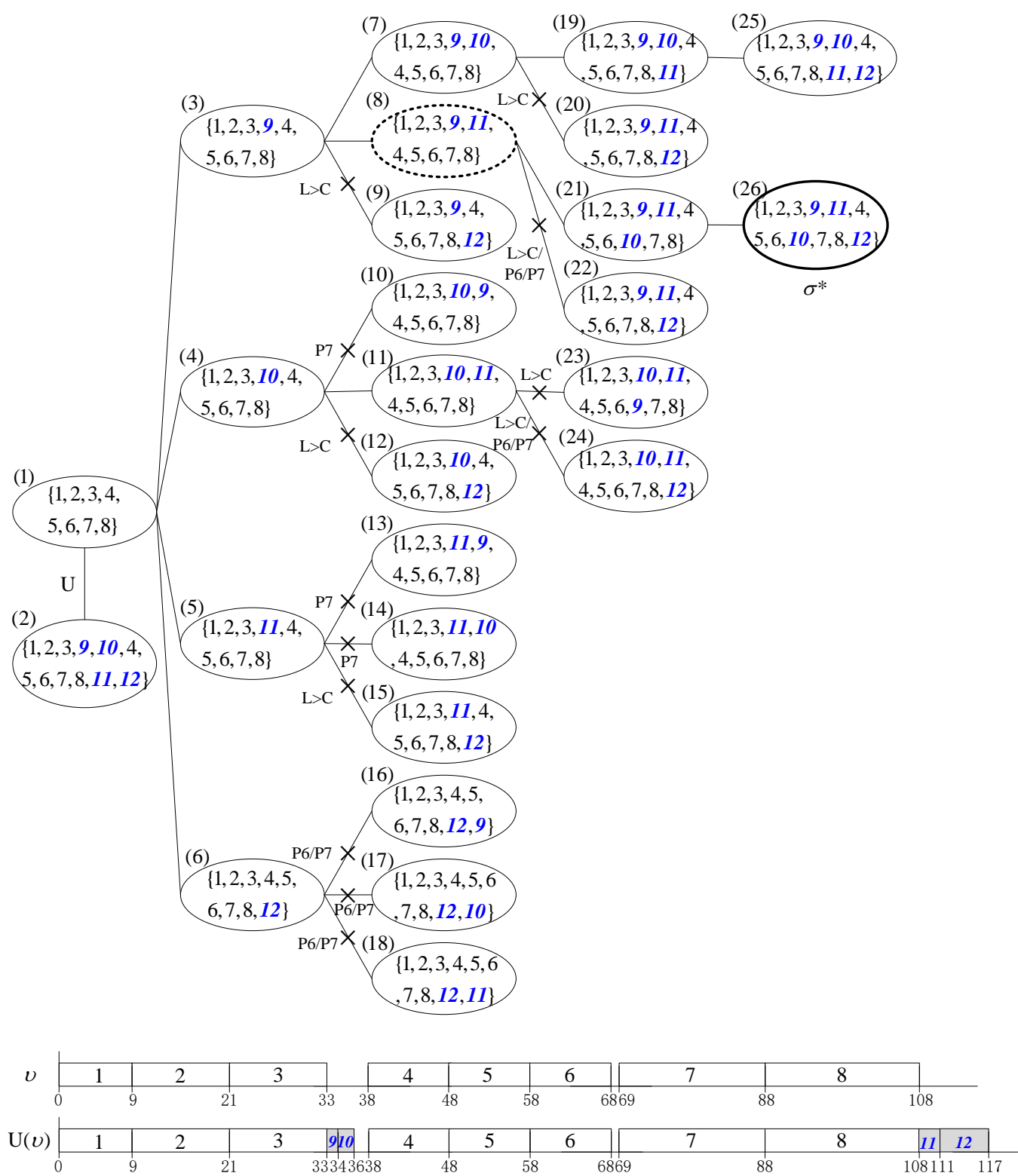

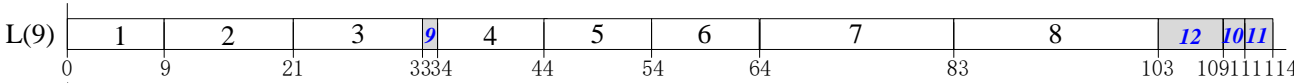

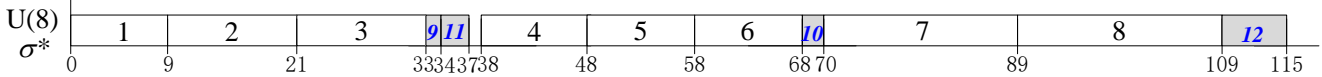

NOTE: The current best solution is updated by U(8). U, L , C and P note upper bound, lower bound, the current best solution and property respectively.

Fig.8 The branch-and-bound for Case I 
Table 1 Seven special case of SRR

\begin{tabular}{|c|c|c|c|c|}
\hline $\begin{array}{l}\text { The theorems } \\
\text { etc. }\end{array}$ & The features of the cases & $\begin{array}{l}\text { The } \\
\text { algorithms }\end{array}$ & $\begin{array}{l}\text { The complexity of the } \\
\text { algorithms }\end{array}$ & The results \\
\hline Theorem 2 & $\begin{array}{l}\mid s_{[j]}(v)+\Delta_{[j]}(v)>C_{[j-1]}(v) \\
+\Delta_{[j-1]}(v) \mid=1, j=1, \ldots, n_{O}\end{array}$ & $\mathrm{KbA}$ & Pseudo-polynomial time & Optimal \\
\hline Theorem 3 & no idle-time in $\sigma$ & $\mathrm{SIH}$ & Polynomial time & Optimal \\
\hline Corollary 1 & $s_{[j]}(\nu)=C_{[j-1]}(\nu), j=1, \ldots, n_{O}$ & $\mathrm{SIH}$ & Polynomial time & Optimal \\
\hline Corollary 2 & $\Delta_{j} \geq \max \left\{p_{i} \mid i \in J_{R}\right\}, j \in J_{O}$ & $\mathrm{SIH}$ & Polynomial time & Optimal \\
\hline Proposition 1 & $p_{j}=p, j \in J_{o}$ & $\mathrm{SIH}$ & Polynomial time & Optimal \\
\hline Proposition 2 & $I_{[1]}+\Delta_{[1]}(v) \geq \sum_{i \in J^{k}} p_{i}$ & $\mathrm{SIH}$ & Polynomial time & Optimal \\
\hline Proposition 3 & $\begin{array}{l}I_{[j]}+\Delta_{[j]}(\nu)<\min \left\{p_{i} \mid i \in J_{R}\right\} \\
{[j] \in J_{O}}\end{array}$ & $\mathrm{SIH}$ & Polynomial time & Optimal \\
\hline Theorem 4 & $I(v)+\Delta_{\left[n_{0}\right]}<\sum_{i \in J_{R}} p_{i}$ & $\mathrm{SIH}$ & Polynomial time & $\begin{array}{l}\text { 3/2-approxi } \\
\text { mation } \\
\text { optimal }\end{array}$ \\
\hline
\end{tabular}

Table 2 Five factors with regard to instants

\begin{tabular}{|c|c|c|c|}
\hline Factor & Description & \multicolumn{2}{|c|}{ Level Meaning } \\
\hline \multirow[t]{2}{*}{$n$} & Problem size, total number of jobs & 20 & Smaller size \\
\hline & & 40 & Bigger size \\
\hline \multirow[t]{3}{*}{$n_{O}$} & Set of rework jobs, total number of rework & $0.25 n$ & Less rework jobs \\
\hline & jobs & $0.5 n$ & $\begin{array}{l}\text { the number of rework jobs equals to of original } \\
\text { jobs }\end{array}$ \\
\hline & & $0.75 n$ & More rework jobs \\
\hline \multirow[t]{2}{*}{$m$} & Size of $p_{i}, i \in J_{R}, \quad p_{i}, i \in J_{R}$ & 20 & $\begin{array}{l}\text { Scheduled with smaller processing times of } \\
\text { rework jobs }\end{array}$ \\
\hline & & 40 & $\begin{array}{l}\text { Scheduled with bigger processing times of rework } \\
\text { jobs }\end{array}$ \\
\hline \multirow[t]{4}{*}{ FIT } & The frequency of idle time within $v$ & 0.1 & Little number of idle-times in original schedule \\
\hline & & 0.3 & $\downarrow$ \\
\hline & & 0.5 & \\
\hline & & 0.7 & More number of idle-times in original schedule \\
\hline \multirow[t]{4}{*}{$\mu$} & a wide range of tightness for $K$ & 0.1 & Little shift allowed for original jobs \\
\hline & & 0.3 & 1 \\
\hline & & 0.5 & 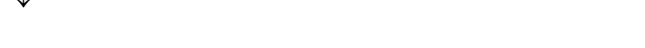 \\
\hline & & 0.7 & large shift allowed for original jobs \\
\hline
\end{tabular}

Table 3 Effect of ${ }_{n}$ on performance of SIH

\begin{tabular}{rrrrrr}
\hline$n$ & NO & CNO & RCNO $(\%)$ & $A E$ & $S D$ \\
\hline 20 & 960 & 905 & 94.27 & 0.001257 & 0.015963 \\
40 & 478 & 459 & 96.03 & 0.000095 & 0.000531 \\
\hline
\end{tabular}


Table 4 Effect of $m$ on performance of SIH

\begin{tabular}{rrrrrr}
\hline$m$ & NO & CNO & RCNO $(\%)$ & $A E$ & $S D$ \\
\hline 20 & 685 & 650 & 94.89 & 0.000706 & 0.007408 \\
40 & 753 & 714 & 94.44 & 0.001021 & 0.016606 \\
\hline
\end{tabular}

Table 5 Effect of $n_{R}$ as a percentage of ${ }_{n}$ on performance of SIH

\begin{tabular}{rrrrrr}
\hline \multicolumn{1}{c}{$n_{R}$} & NO & CNO & RCNO $(\%)$ & $A E$ & $S D$ \\
\hline $0.25 n$ & 640 & 632 & 98.59 & 0.000549 & 0.008348 \\
$0.5 n$ & 427 & 407 & 95.20 & 0.001177 & 0.019911 \\
$0.75 n$ & 371 & 325 & 87.60 & 0.001075 & 0.009211 \\
\hline
\end{tabular}

Table 6 Effect of FIT on performance of SIH

\begin{tabular}{lrrrrr}
\hline FIT & NO & CNO & RCNO $(\%)$ & $A E$ & $S D$ \\
\hline 0.1 & 371 & 363 & 97.84 & 0.000042 & 0.000307 \\
0.3 & 355 & 336 & 94.65 & 0.001855 & 0.023659 \\
0.5 & 360 & 333 & 92.50 & 0.000563 & 0.003230 \\
0.7 & 352 & 332 & 94.32 & 0.001675 & 0.109854 \\
\hline
\end{tabular}

Table 7 Effect of $\mu$ on performance of SIH

\begin{tabular}{rrrrrr}
\hline$\mu$ & $\mathrm{NO}$ & $\mathrm{CNO}$ & $\mathrm{RCNO}(\%)$ & \multicolumn{1}{c}{$A E$} & $S D$ \\
\hline 0.1 & 396 & 324 & 81.82 & 0.002700 & 0.023219 \\
0.3 & 346 & 345 & 99.71 & 0.000029 & 0.000547 \\
0.5 & 338 & 338 & $\mathbf{1 0 0 . 0 0}$ & 0 & 0 \\
0.7 & 358 & 357 & 99.72 & 0.000484 & 0.009165 \\
\hline
\end{tabular}

Table 8 information of jobs for Case I

\begin{tabular}{ccccccccccccc}
\hline$i$ & 1 & 2 & 3 & 4 & 5 & 6 & 7 & 8 & 9 & 10 & 11 & 12 \\
\hline$p_{i}$ & 9 & 12 & 12 & 9 & 10 & 10 & 19 & 20 & 1 & 2 & 3 & 6 \\
$r_{i}$ & 0 & 0 & 1 & 38 & 38 & 38 & 69 & 69 & 0 & 0 & 0 & 0 \\
\hline
\end{tabular}




\section{Acknowledgments}

This work is supported by the National Science Foundation for Distinguished Young Scholars of China under Grant No. 71325002, No. 61225012; the National Natural Science Foundation of China under Grant No. 71071028, No. 71171040, No. 21506014; the Fundamental Research Funds for State Key Laboratory of Synthetical Automation for Process Industries under Grant No. $2013 Z C X 11$. 